\title{
Differences in the sensitivity of ovarian cancer to photodynamic therapy and the mechanisms for those differences
}

\author{
YOSHIHITO YOKOYAMA, TATSUHIKO SHIGETO, RIE MIURA, ASAMI KOBAYASHI, \\ MAKITO MIZUNUMA, AISA YAMAUCHI, MASAYUKI FUTAGAMI and HIDEKI MIZUNUMA \\ Department of Obstetrics and Gynecology, Hirosaki University Graduate School of Medicine, \\ Hirosaki, Aomori 036-8562, Japan
}

Received December 12, 2015; Accepted February 17, 2017

DOI: $10.3892 / \mathrm{ol} .2017 .6095$

\begin{abstract}
Protoporphyrin IX (PpIX) levels are crucial to the antitumor action of photodynamic therapy (PDT). In the present study, the underling molecular mechanisms for the variation in PpIX levels in ovarian cancer cells were investigated. Five ovarian cancer cell lines were subcutaneously grafted onto the backs of nude mice. Once tumors had developed, 5-aminolevulinic acid methyl ester hydrochloride (methyl-ALA) was administered intraperitoneally and the tumor was irradiated twice/week. PpIX levels in the tumor were assayed using high-performance liquid chromatography. Enzymes involved in heme synthesis and degradation were screened using a microarray technique. Expression of the glutathione transferase Omega-1 (GSTO1) gene involved in the conversion of PpIX into heme in cells was quantified using the reverse transcription-quantitative polymerase chain reaction. In HTOA, HRA and DISS cells, PDT resulted in significant tumor shrinkage in comparison with the controls. In MCAS and TOV21G cells, no significant alterations in tumor growth were identified compared with the untreated cells. PpIX levels increased significantly in HTOA, DISS and HRA cells compared with in MCAS and TOV21G cells. A comparison of genetic profiles using PDT-sensitive DISS cells and PDT-resistant MCAS cells indicated that MCAS cells exhibited significantly increased levels of $\delta$-aminolevulinate synthase (a rate-limiting enzyme in heme synthesis), heme oxygenase 2 (an enzyme that degrades heme into biliverdin), and biliverdin reductase $B$ (an enzyme that reduces biliverdin into bilirubin) in comparison with DISS cells. The level of GSTO1 expression in HTOA, HRA and DISS cells was 2.5 -fold that in MCAS and TOV21G cells. Sensitivity
\end{abstract}

Correspondence to: Dr Yoshihito Yokoyama, Department of Obstetrics and Gynecology, Hirosaki University Graduate School of Medicine, 5-Zaifu-cho, Hirosaki, Aomori 036-8562, Japan

E-mail: yokoyama@hirosaki-u.ac.jp

Key words: photodynamic therapy, 5-aminolevulinic acid methyl ester hydrochloride, protoporphyrin IX, glutathione transferase Omega-1, ovarian cancer to PDT is related to PpIX levels in cells. The results of the present study suggested that PpIX tends not to accumulate in PDT-resistant cells despite active heme synthesis and degradation, and that high levels of GSTO1 expression are associated with increased sensitivity to PDT.

\section{Introduction}

The standard treatment for ovarian cancer is surgery followed by anticancer therapy (1). A total of $70 \%$ of advanced ovarian cancer recurs, so advanced ovarian cancer ultimately has a 5-year survival rate of between 30 and $40 \%$ (2). Ovarian cancer has the highest mortality rate among gynecological malignancies (3).

Photodynamic therapy (PDT) has garnered attention as a novel therapy to reduce the tumor burden on a patient. PDT has been used to treat superficial esophageal cancer, early lung cancer and early gastric cancer (4-6). In gynecology, PDT has been used to treat early cancer of the cervix (7). Younger patients tend to opt to receive treatment that preserves fertility, and PDT may be such a treatment (7). Therefore, attention has focused on the usefulness of PDT in that regard. Unlike conventional approaches, PDT leaves the cervix intact, has a high cure rate, and does not hamper pregnancy or delivery following surgery (7).

PDT is a therapy involving photosensitizers (or their precursors) with an affinity for tumors. The specific accumulation of photosensitizers in tumor tissue and blood vessels and the excitation of light are used by PDT to produce singlet oxygen (a type of reactive oxygen species) with potent cytocidal action. PDT using hematoporphyrin derivatives to treat skin metastasis of breast cancer was first studied by Dougherty et al (8) in 1979. Subsequently, PDT has been investigated in numerous studies. PDT does not substantially damage normal tissue and it treats lesions with low levels of light energy, so various lesions may be treated by irradiation using a laser beam. Previously, we have described the potential effectiveness of using PDT to treat ovarian cancer; however, certain types of ovarian cancer are resistant to PDT (9). Results suggested that protoporphyrin IX (PpIX) in cells is crucial to satisfactory treatment of cancer with PDT (9). PpIX is a metabolite that is converted from aminolevulinic acid via heme synthesis and degradation pathways (10). 
In the present study, five ovarian cancer cell lines with distinct histological types were used to produce subcutaneous tumors in mice. Subsequently, the effectiveness of PDT in treating those tumors was determined. In addition, PDT-sensitive cells and therapy-resistant cells were identified, and a microarray technique was used to compare the expression of genes that code for enzymes involved in heme synthesis and degradation. Glutathione transferase Omega 1 (GSTO1) is involved in the conversion of PpIX into heme. The level of expression of GSTO1 was compared in the various cell lines.

\section{Materials and methods}

Cell lines and cell culture. HTOA cells were obtained from RIKEN BioResource Center (Tsukuba, Japan). MCAS and TOV21G cells were obtained from the American Type Culture Collection (Manassas, VA, USA). HRA and DISS cells were provided by Dr Y. Kikuchi (National Defense Medical College, Tokorozawa, Japan) (11) and Dr Y. Saga (Jichi Medical School, Tochigi, Japan), respectively. HTOA, HRA and DISS cells were derived from human ovarian serous adenocarcinoma. MCAS and TOV21G cells were derived from human ovarian mucinous and clear cell adenocarcinoma, respectively. These five cell lines were verified in writing as being ovarian in origin. All cell lines were grown in RPMI-1640 medium with $10 \%$ fetal bovine serum, 100 units $/ \mathrm{ml}$ penicillin and $100 \mu \mathrm{g} / \mathrm{ml}$ streptomycin at $37^{\circ} \mathrm{C}$ in a humidified atmosphere containing $5 \% \mathrm{CO}_{2} / 95 \%$ air.

Animals. Animal experiments were approved by the Animal Research Committee of Hirosaki University (Hirosaki, Japan) and all animals were cared for and handled in accordance with the Rules for Animal Experimentation of Hirosaki University and animal practices as defined by national and local bodies governing animal welfare (Guide for the Care and Use of Laboratory Animals published by the National Institutes of Health). A total of 10 8-week-old female BALB/c nu/nu mice weighing 20-25 g (CLEA Japan, Inc., Tokyo, Japan) were used in the present study. All mice were group housed in plastic cages with stainless steel grid tops in an air-conditioned room with a 12-h light/12-h dark cycle and fed with water and food ad libitum in the Institute for Animal Experiments of Hirosaki University.

Cancer-bearing mouse model. Cancer cells $\left(0.5 \times 10^{6}\right.$ cells $)$ in $200 \mu \mathrm{l}$ PBS were injected subcutaneously into the dorsal region of the nude mice under general anesthesia. Tumors were grown until the largest diameter reached $3 \mathrm{~mm}$ prior to commencing treatment. Then, the mice were divided into two groups, a treatment group and a control group, containing 5 mice each. The tumor dimensions were measured three times/week using a caliper and tumor volume was calculated using the equation $\mathrm{V}\left(\mathrm{mm}^{3}\right)=\mathrm{AxB}^{2} / 2$, where $\mathrm{A}$ is the largest diameter and $\mathrm{B}$ is the smallest diameter.

PDT. 5-Aminolevulinic acid methyl ester hydrochloride (methyl-ALA) was obtained from Cosmo Bio International (Tokyo, Japan). Immediately following dissolution in PBS at a concentration of $10 \mathrm{mg} / \mathrm{ml}$, PBS. The methyl-ALA solution was injected intraperitoneally into the nude mice of the treatment group. The mice in the treatment group and in the control group received an intraperitoneal injection of $250 \mathrm{mg} / \mathrm{kg}$ methyl-ALA and PBS alone, respectively (9). PDT was administered by 10 min irradiation using a $150 \mathrm{~W}$ halogen light, $3 \mathrm{~h}$ after methyl-ALA or PBS injection (control) (9). Each mouse received PDT twice/week for 3 weeks.

Determination of intracellular PpIX following methyl-ALA exposure. The cellular uptake of methyl-ALA, which was converted into fluorescent PpIX inside HTOA, HRA, DISS, MCAS and TOV21G cells, was determined using high-performance liquid chromatography (HPLC). Each cell line was seeded in 100-mm culture dishes and incubated with $5.5 \mu \mathrm{M}$ freshly prepared methyl-ALA for $3 \mathrm{~h}$. Methyl-ALA was removed and the cells were trypsinized and centrifuged at $10,000 \mathrm{x} \mathrm{g}$ for $5 \mathrm{~min}$ at $4^{\circ} \mathrm{C}$, then washed twice with ice-cold PBS. The cell pellet was then resuspended in $1 \mathrm{ml}$ radioimmunoprecipitation assay buffer (Sigma-Aldrich; Merck KGaA; Darmstadt, Germany). The mixture was agitated gently for $15 \mathrm{~min}$ on ice and centrifuged at $10,000 \mathrm{x} \mathrm{g}$ for $5 \mathrm{~min}$ at $4^{\circ} \mathrm{C}$ to pellet the cell debris. The supernatant was transferred to a new tube and subjected to HPLC analysis (LC-20AT Prominence; Shimadzu, Kyoto, Japan) equipped with a reversed-phase C18 column (CAPCELL PAK, C18; SG300; $5 \mu \mathrm{m}, 4.6 \times 250 \mathrm{~mm}$; Shiseido, Tokyo, Japan). Elution was started with $10 \%$ solvent A (50 mM phosphate buffer) and $90 \%$ solvent B (acetonitrile) for $7 \mathrm{~min}$. The elution flow throughout was kept constant at a rate of $2.0 \mathrm{ml} / \mathrm{min}$. The excitation wavelength was set at $404 \mathrm{~nm}$ and the fluorescence emission wavelength was set at $620 \mathrm{~nm}$. Subsequent to dissolving $10 \mathrm{mg}$ of PpIX disodium salt (Sigma-Aldrich; Merck KGaA) with a few drops of $1 \mathrm{M} \mathrm{HCl}$, a stock PpIX standard was prepared by diluting it in $50 \mathrm{ml}$ of N,N-dimethylformamide. All results are presented for three independent experiments.

Total RNA isolation. Cells were homogenized using a homogenizer and RNA was extracted using TRIzol ${ }^{\circledR}$ reagent (Invitrogen; Thermo Fisher Scientific, Inc., Waltham, MA, USA), according to the manufacturer's protocol. The extracted total RNA was then purified using an RNeasy MiniElute Cleanup kit column (Qiagen, Inc., Valencia, CA, USA) with incubation with DNase (Qiagen, Inc.). Total RNA extracted was quantified using an Eppendorf UV spectrophotometer, and the integrity of the RNA samples was monitored using an Agilent 2100 Bioanalyzer (Agilent Technologies, Inc., Santa Clara, CA, USA) and a NanoDrop ND-1000 kit (Thermo Fisher Scientific, Inc.). Only RNAs with an $\mathrm{A}_{260} / \mathrm{A}_{280}$ ratio $>1.8$ and an RNA integrity number $>7$ were used for microarray experiments. The remaining good-quality RNAs were retained for subsequent confirmative reverse transcription-quantitative polymerase chain reaction (RT-qPCR) experiments.

Microarray analysis. Each good-quality sample was hybridized to the Affymetrix HG-U133 plus 2.0 GeneChip (Affymetrix, Inc., Santa Clara, CA, USA). This gene chip analyzes the expression level of 38,500 well-characterized human genes. First, $10 \mu \mathrm{g}$ of the total RNA was reverse-transcribed with the SuperScript Choice System (Invitrogen; Thermo Fisher Scientific, Inc.) with oligo dT primers containing a T7 RNA polymerase promoter site. cDNA was 
then in vitro-transcribed and labeled with biotin using the IVT labeling kit (Affymetrix, Inc.), followed by the fragmentation of the biotinylated cRNA. The quality of this cRNA was then assessed with the Agilent 2100 Bioanalyzer (Agilent Technologies, Inc.). The fragmented cRNA was hybridized overnight to Affymetrix Human Genome U133APlus 2.0 Arrays (Affymetrix, Inc.) and scanned according to the manufacturer's guidelines. The chips were washed and stained using the GeneChip Fluidics Station 400 (Affymetrix, Inc.) and then scanned with the GeneChip Scanner 3000 (Affymetrix, Inc.). Labeling, hybridization, image scanning and data analysis were performed by Kurabo Industries Ltd. (Osaka, Japan).

$R T$ - $q P C R$. qPCR was used to evaluate the expression of the GSTO1 gene. TaqMan ${ }^{\circledR}$ Gene Expression assays (Thermo Fisher Scientific, Inc.) was used with TaqMan ${ }^{\circledR}$ probes for GSTO1 and $\beta$-actin $(A C T B)$. Primers used for PCR were as follows: GSTO1, 5'-AGGATCCACGATGTCCGGGGAGTC AG-3' (forward) and 5'-CGAATTCAGAGCCCATAGTCA CAG-3' (reverse), and ACTB, 5'-AGTCCCTTGCCATCCTAA AAGC-3' (forward) and 5'-GGGAGAGGACTGGGCCAT T-3' (reverse). Prior to qPCR, reverse transcription of $500 \mathrm{ng}$ total RNA was performed using a SuperScript ${ }^{\circledR}$ VILO $^{\mathrm{TM}}$ cDNA Synthesis kit (Thermo Fisher Scientific, Inc.), according to the manufacturer's protocol. qPCRs were set up in $20 \mu \mathrm{l}$ volumes with 50-fold cDNA dilutions, 20X TaqMan ${ }^{\circledR}$ gene expression assay mixture, $2 \mathrm{X}$ TaqMan ${ }^{\circledR}$ Universal PCR master mix II (Thermo Fisher Scientific, Inc.) and distilled water. PCRs were performed in quadruplicate following the manufacturer's protocols on a model ABI Prism 7000 Real-Time PCR system (Thermo Fisher Scientific, Inc.) using the following protocol. Initial denaturation and polymerase activation at $95^{\circ} \mathrm{C}$ for $10 \mathrm{~min}$, followed by 40 cycles of denaturation at $95^{\circ} \mathrm{C}$ for $15 \mathrm{sec}$ and annealing and extension at $60^{\circ} \mathrm{C}$ for $1 \mathrm{~min}$. ACTB was used as a reference gene for normalization of samples.

Statistical analysis. The results are presented as the mean \pm standard deviation. Differences in tumor volume between the groups were analyzed using a non-parametric Mann-Whitney U test. Other statistical analyses were carried out using Student's t-test, $\chi^{2}$ test or Fisher's exact probability test. $\mathrm{P}<0.05$ was considered to indicate a statistically significant difference.

\section{Results}

Antitumor action of PDT in ovarian cancer cell lines. PDT resulted in significant tumor shrinkage in HTOA, HRA and DISS tumor cells in comparison with the controls $(\mathrm{P}<0.05$; Fig. 1). No significant differences in tumor volume were identified in MCAS and TOV21G cells between the PDT group and the control group (Fig. 1).

PpIX levels in ovarian cancer cells. Methyl-ALA is converted into PpIX. PpIX levels increased significantly in HTOA, DISS and HRA cells in comparison with levels in MCAS and TOV21G cells (Table I).

Genetic profile of enzymes involved in heme synthesis and degradation in DISS and MCAS cells. The genetic profile of
Table I. Determination of protoporphyrin IX in five ovarian cancer cell lines.

\begin{tabular}{lc}
\hline Cell line & Amount of protoporphyrin IX, mg/dl \\
\hline HTOA & $62.6 \pm 9.4^{\mathrm{a}}$ \\
HRT & $67.7 \pm 2.7^{\mathrm{a}}$ \\
DISS & $59.3 \pm 8.9^{\mathrm{a}}$ \\
MCAS & $22.4 \pm 4.7$ \\
TOV21G & $24.1 \pm 3.9$ \\
\hline
\end{tabular}

${ }^{\mathrm{a}} \mathrm{P}<0.005$ vs. MCAS and TOV21G cells.
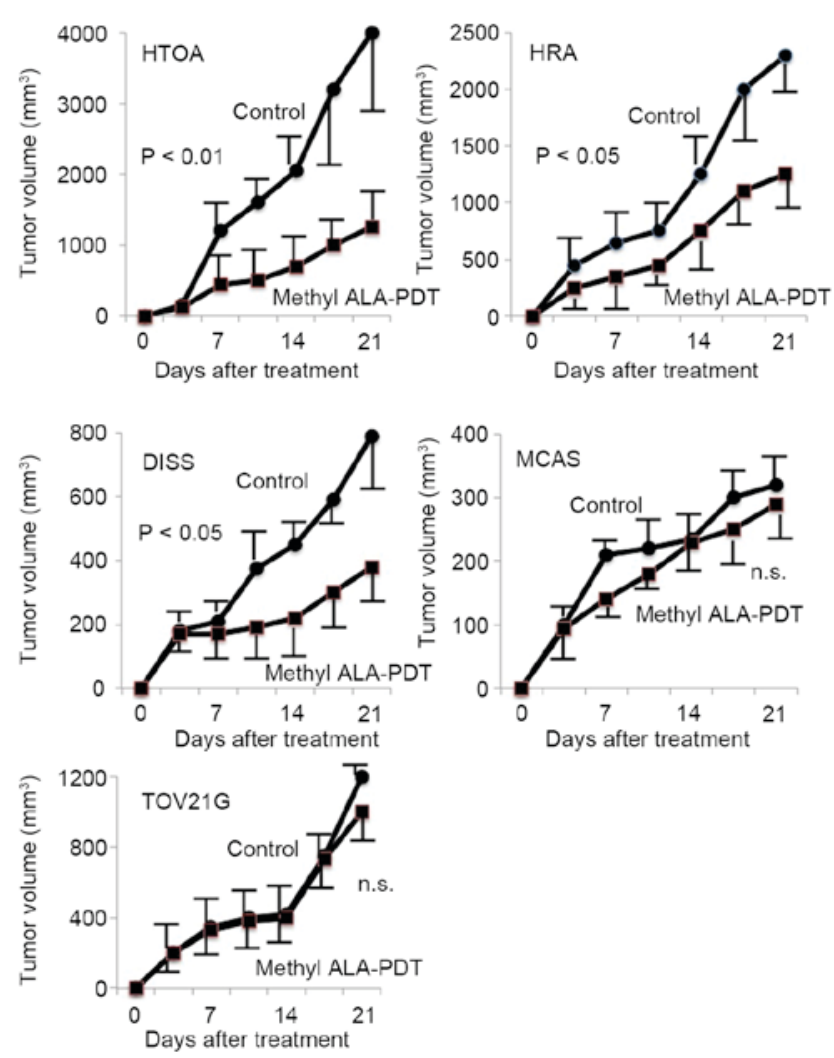

Figure 1. Antitumor effect of methyl-ALA-PDT on tumor derived from five ovarian cancer cell lines. In HTOA, HRA and DISS tumor cells, PDT resulted in significant tumor shrinkage in comparison with the untreated control cells. In MCAS and TOV21G cells, no significant differences in tumor growth were identified between the PDT group and the control cells.

enzymes involved in heme synthesis and degradation (Fig. 2) was determined using microarray assay. A 3-fold increase in the level of expression was defined as marked upregulation (Table II). $\delta$-aminolevulinate ( $\delta$-ALA) synthase is a rate-limiting enzyme in heme synthesis (Fig. 2), and expression of $\delta$-ALA synthase was increased markedly in MCAS cells in comparison with that in DISS cells (Table II). Heme oxygenase 2 is an enzyme that degrades heme into biliverdin and biliverdin reductase $\mathrm{B}$ is an enzyme that reduces biliverdin into bilirubin (Fig. 2). The expression levels of heme oxygenase 2 and biliverdin reductase B increased 11.5-fold and 6.8-fold, respectively, in MCAS cells in comparison with levels of expression in DISS cells (Table II). 
Table II. Results of microarray analysis on the expression of enzymes involved in the heme synthetic and degradation pathway in DISS and MCAS cells.

Signal

Gene title

DISS

MCAS

Fold difference (MCAS/DISS)

Heme synthetic pathway

$\delta$-aminolevulinic synthase

Hydroxymethylbilane synthase

1,040

830

Uroporphyrinogen III synthase

Uroporphyrinogen decarboxylase

Coproporphyrinogen III oxidase

Protoporphyrinogen oxidase

Ferrochelatase

Heme degradation pathway

Heme oxygenase 1

Heme oxygenase 2

Biliverdin reductase $\mathrm{B}$
1,010

4,710

2,220

690

500

150

90

540
3,380

770

1,530

2,320

1,620

510

250

310

1,040

3,680
3.25

0.93

1.51

0.49

0.73

0.74

0.50

2.07

11.56

6.81

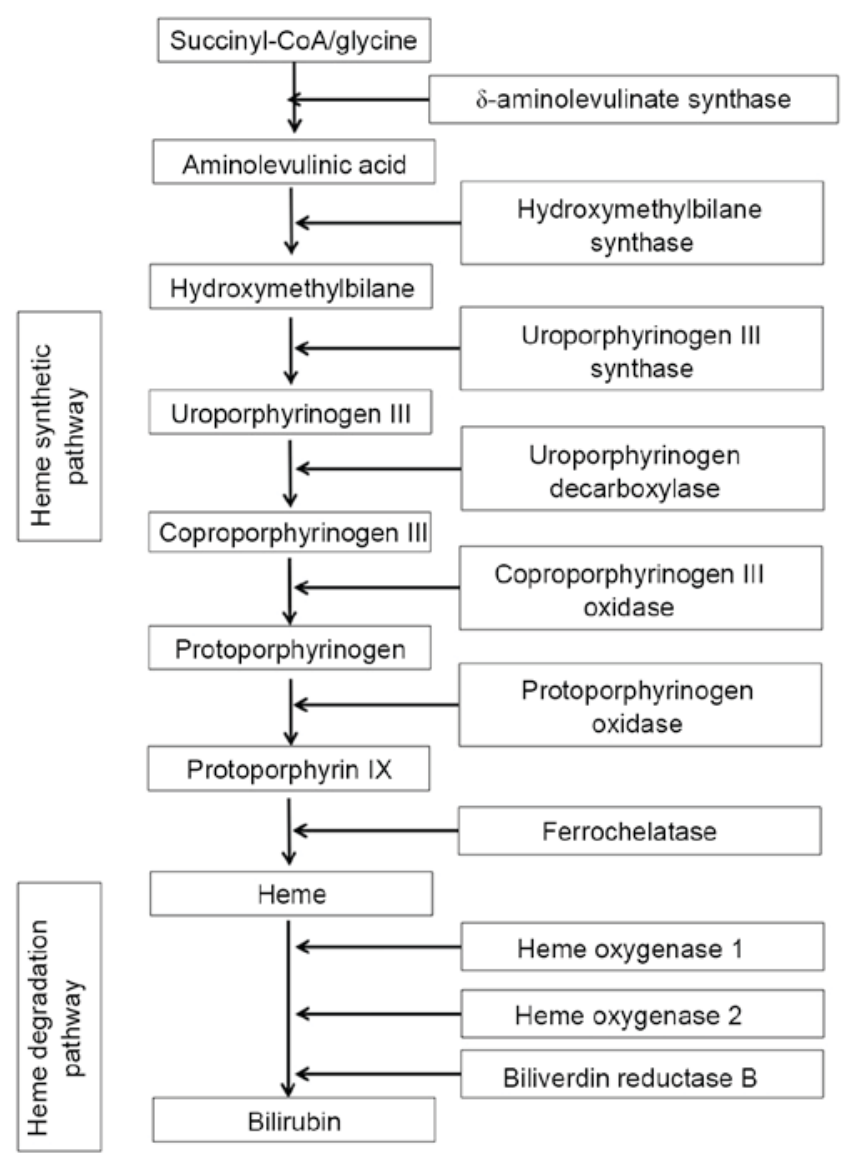

Figure 2. Schematic diagram of the heme synthetic and degradation pathways.

Expression of GSTO1 in ovarian cancer cells. The expression level of GSTO1 in HTOA, HRA and DISS cells was 2.5 -fold increased compared with that in either MCAS or TOV21G cells, a difference which was determined to be significant ( $\mathrm{P}<0.001$; Fig. 3).

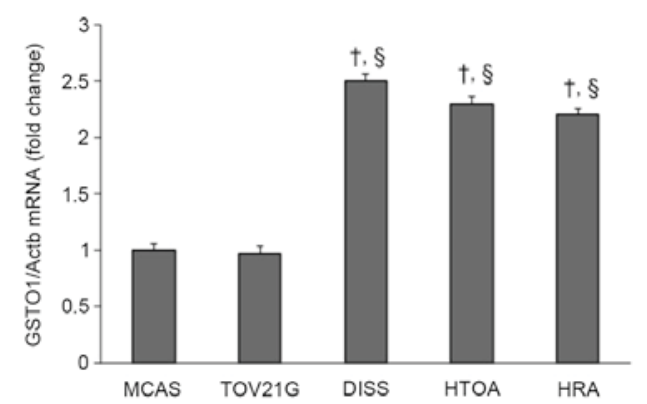

Figure 3. Determination of the GSTO1 gene in ovarian cancer cells. The expression level of GSTO1 in HTOA, HRA and DISS cells was between 2 and 2.5 -fold that in MCAS and TOV21G cells. ${ }^{\top} \mathrm{P}<0.001$ vs. MCAS cells; ${ }^{\S} \mathrm{P}<0.001$ vs. TOV21G. GSTO1, glutathione transferase Omega-1; Actb, $\beta$-actin.

\section{Discussion}

During PDT, methyl-ALA is taken up by cells and converted into PpIX. PpIX levels are a crucial aspect of the antitumor action of PDT. In the present study, it was identified that antitumor action various depending on PpIX levels in cells. In other words, PDT is markedly more effective at treating tumors developing from ovarian cancer cells with high PpIX levels.

PpIX is an intermediate in heme synthesis and degradation (10). When $5.5 \mu \mathrm{M}$ methyl-ALA was added to ovarian cancer cells, PDT-sensitive HTOA, HRA and DISS cells exhibited significantly higher PpIX levels in comparison with PDT-resistant MCAS and TOV21G cells. A microarray assay was used to analyze the genetic profile of converting enzymes involved in heme synthesis and degradation in DISS and MCAS cells. This analysis indicated that MCAS cells exhibited increased expression (3-fold) of the $\delta$-ALA synthase gene (which codes for a rate-limiting enzyme in heme synthesis), increased expression (10-fold) of the heme oxygenase 2 gene (which codes for an enzyme that degrades 
heme into biliverdin), and increased expression (7-fold) of the biliverdin reductase $\mathrm{B}$ gene (which codes for an enzyme that reduces biliverdin into bilirubin) in comparison with DISS cells. These results suggest that PpIX tends not to accumulate in PDT-resistant cells despite active heme synthesis and degradation, and despite the uptake of a derivative of an exogenous amino acid.

Sustaining PpIX levels in cells is an aspect that requires consideration in order to increase the effectiveness with which PDT is able to treat ovarian cancer. Ferrochelatase is an enzyme that converts PpIX into heme. Inhibiting ferrochelatase may assist in sustaining PpIX levels in cells. Lead is a typical ferrochelatase inhibitor; however, the use of such an inhibitor clinically would be precluded since lead poisoning causes porphyria (12). The inhibition of heme oxygenase increases the accumulation of heme, i.e. it increases the levels of iron, a potent source of free radicals (13).

GSTO1 inhibits the conversion of PpIX into heme (14) and GSTO1 catalyzes the detoxification of heavy metals including iron (through the conjugation of glutathione to those substances) (15). Heme induces expression of the erythropoietin gene through activation of hypoxia-inducible factor 1 (16). Erythropoietin is a hematopoietic hormone, and erythropoietin is known to inhibit sensitivity to PDT (17). Recent studies have revealed that tyrosine protein kinase Met (c-Met)/phosphoinositide 3-kinase (PI3K) signaling is related to resistance to PDT (18). Erythropoietin may induce resistance to PDT through the activation of c-Met/PI3K signaling. GSTO1 inhibits the conversion of PpIX into heme, so GSTO1 may act to sustain PpIX levels in cells and inhibit expression of the erythropoietin gene, thus assisting with the maintenance of sensitivity to PDT. In the present study, the expression of GSTO1 in DSS cells increased significantly in comparison with that in MCAS cells. This result is consistent with the result that DISS cells exhibited significantly higher PpIX levels compared with MCAS cells.

PDT is accompanied by oxidative stress (19). Oxidative stress is a biological phenomenon whereby reactive oxygen species are produced and subsequently cause cellular damage. In cancer tissue, inflammation has been triggered and angiogenesis is extensive, therefore further reactive oxygen species are produced. A recent study identified that GSTO1 provides protection from oxidative stress (20). This result is consistent with the results of the present study that DISS cells that expressed increased levels of GSTO1 responded well to PDT.

The photosensitizer precursor used in the present study was methyl-ALA. Methyl-ALA has been demonstrated to be selectively taken up by tumor tissue (9). Other approaches are being investigated, including delivery of a photosensitizer to a tumor via a nanoparticle drug delivery system (21) and increasing tumor selectivity by linking a photosensitizer to the gene encoding $\beta$-galactosidase (22). Studies by the present authors are underway to examine transfer of the GSTO1 gene to tumor cells and direct induction of PpIX in tumor cells. Further research is warranted to ensure that PDT is universally effective at treating a range of cancer cells.

Sensitivity to PDT is associated with PpIX levels in cells. The results of the present study suggested that PpIX tends not to accumulate in PDT-resistant cells despite active heme synthesis and degradation and despite the uptake of a derivative of an exogenous amino acid. The results of the present study suggested that high levels of GSTO1 expression are associated with increased sensitivity to PDT.

\section{Acknowledgements}

The present study was supported by a Grant-in Aid for Cancer Research from the Ministry of Education, Science and Culture of Japan (grant no. 20591935 to Y.Y.).

\section{References}

1. Hofstetter G, Concin N, Braicu I, Chekerov R, Sehouli J, Cadron I, Van Gorp T, Trillsch F, Mahner S, Ulmer H, et al: The time interval from surgery to start of chemotherapy significantly impacts prognosis in patients with advanced serous ovarian carcinoma-analysis of patient data in the prospective OVCAD study. Gynecol Oncol 131: 15-20, 2013.

2. Akeson M, Zetterqvist BM, Dahllöf K, Brännström M and Horvath G: Effect of adjuvant paclitaxel and carboplatin for advanced stage epithelial ovarian cancer: A population-based cohort study of all patients in western Sweden with long-term follow-up. Acta Obstet Gynecol Scand 87: 1343-1352, 2008.

3. Siegel R, Naishadham D and Jemal A: Cancer statistics, 2013. CA Cancer J Clin 63: 11-30, 2013.

4. Casson AG: Photofrin PDT for early stage esophageal cancer: A new standard of care? Photodiagnosis Photodyn Ther 6: 155-156, 2009.

5. Ikeda N, Usuda J, Kato H, Ishizumi T, Ichinose S, Otani K, Honda H, Furukawa K, Okunaka T and Tsutsui H: New aspects of photodynamic therapy for central type early stage lung cancer. Lasers Surg Med 43: 749-754, 2011.

6. Spinelli P, Dal Fante M and Mancini A: Current role of laser and photodynamic therapy in gastrointestinal tumors and analysis of a 10-year experience. Semin Surg Oncol 8: 204-213, 1992.

7. Choi MC, Lee C and Kim SJ: Efficacy and safety of photodynamic therapy for cervical intraepithelial neoplasia: A systemic review. Photodiagnosis Photodyn Ther 11: 479-480, 2014.

8. Dougherty TJ, Lawrence G, Kaufman JH, Boyle D, Weishaupt KR and Goldfarb A: Photoradiation in the treatment of recurrent breast carcinoma. J Natl Cancer Inst 62: 231-237, 1979.

9. Wakui M, Yokoyama Y, Wang H, Shigeto T, Futagami M and Mizunuma H: Efficacy of a methyl ester of 5-aminolevulinic acid in photodynamic therapy for ovarian cancers. J Cancer Res Clin Oncol 136: 1143-1150, 2010.

10. Furuyama K, Harigae H, Kinoshita C, Shimada T, Miyaoka K, Kanda C, Maruyama Y, Shibahara S and Sassa S: Late-onset $\mathrm{X}$-linked sideroblastic anemia following hemodialysis. Blood 101: 4623-4624, 2003.

11. Kikuchi Y, Kizawa I, Oomori K, Miyauchi M, Kita T, Sugita M, Tenjin Y and Kato K: Establishment of a human ovarian cancer cell line capable of forming ascites in nude mice and effects of tranexamic acid on cell proliferation and ascites formation. Cancer Res 47: 592-596, 1987.

12. Wetterberg L: Acute porphyria and lead poisoning. Lancet 1: 498, 1966.

13. Ho PS, Hoffman BM, Kang CH and Margoliash E: Control of the transfer of oxidizing equivalents between heme iron and free radical site in yeast cytochrome c peroxidase. J Biol Chem 1258: 4356-4363, 1983.

14. Kodym R, Calkins PR and Story MD: Anthracycline-induced erythroid differentiation of K562 cells is inhibited by p28, a novel mammalian glutathione-binding stress protein. Leuk Res 25: 151-156, 2001

15. Paiva L, Hernández A, Martínez V, Creus A, Quinteros D and Marcos R: Association between GSTO2 polymorphism and the urinary arsenic profile in copper industry workers. Environ Res 110: 463-468, 2010.

16. Hofer T, Wenger RH, Kramer MF, Ferreira GC and Gassmann M: Hypoxic up-regulation of erythroid 5-aminolevulinate synthase. Blood 101: 348-350, 2003.

17. Solár P, Koval J, Mikes J, Kleban J, Solárová Z, Lazúr J, Hodorová I, Fedorocko P and Sytkowski AJ: Erythropoietin inhibits apoptosis induced by photodynamic therapy in ovarian cancer cells. Mol Cancer Ther 7: 2263-2271, 2008. 
18. Jung KA, Choi BH and Kwak MK: The c-MET/PI3K signaling is associated with cancer resistance to doxorubicin and photodynamic therapy by elevating BCRP/ABCG2 expression. Mol Pharmacol 87: 465-476, 2015.

19. Luna MC, Wong S and Gomer CJ: Photodynamic therapy mediated induction of early response genes. Cancer Res 54: 1374-1380, 1994.

20. Meng F, Zhang Y, Liu F, Guo X and Xu B: Characterization and mutational analysis of omega-class GST (GSTO1) from Apis cerana cerana, a gene involved in response to oxidative stress. PLoS One 9: e93100, 2014
21. Li Z, Sun L, Lu Z, Su X, Yang Q, Qu X, Li L, Song K and Kong B: Enhanced effect of photodynamic therapy in ovarian cancer using a nanoparticle drug delivery system. Int J Oncol 47: 1070-1076, 2015.

22. Koide Y, Urano Y, Yatsushige A, Hanaoka K, Terai T and Nagano T: Design and development of enzymatically activatable photosensitizer based on unique characteristics of thiazole orange. J Am Chem Soc 131: 6058-6059, 2009. 